\title{
DARBOUX RETRACTS
}

\author{
FRED ROUSH, RICHARD G. GIBSON AND KENNETH R. KELLUM
}

\begin{abstract}
Darboux retracts are defined, and it is proved that any continuum contained in a separable metric space is a Darboux retract. This leads immediately to a very general extension theorem.
\end{abstract}

A subspace $A$ of a space $X$ is said to be a retract of $X$ if there exists a continuous function (called a retraction) $r: X \rightarrow A$ such that $r(x)=x$ for each $x \in A$. In recent years several authors have studied generalized retracts in which the retraction function is allowed to be discontinuous, [3], [4], [6], [7], [8]. In the present paper, motivated primarily by the work of Cornette on connectivity retracts, we study the less restrictive class of Darboux retracts. Despite the similarity in the definition of Darboux and connectivity functions, retracts under these two types of functions differ markedly. Cornette has proved that a connectivity retract of a unicoherent Peano continuum is a unicoherent Peano continuum. On the other hand, we show that any continuum contained in a separable metric space $X$ is a Darboux retract of $X$.

A function $f: X \rightarrow Y$ is connectivity if $f \mid C$ is a connected subset of $X \times Y$ for each connected subset $C$ of $X$. A function $f: X \rightarrow Y$ is Darboux if $f(C)$ is a connected subset of $Y$ for each connected subset $C$ of $X$. For a survey on Darboux functions see [2].

EXAMPLe 1. An example of a Darboux retract of $R^{2}$ which is neither closed nor locally connected. Let $A=\{(x, \sin (1 / x)): 0<x<1 / \pi\} \cup\{(0,0)\}$ and define $f$ : $R^{2} \rightarrow A$ by

$$
f(x, y)= \begin{cases}(1 / \pi, 0) & \text { if } x>1 / \pi \\ (0,0) & \text { if } x<0 \\ (x, \sin (1 / x)) & \text { if } 0<x<1 / \pi\end{cases}
$$

Example 2. Similarly, the closure of the set $A$ of Example 1 is a closed but not locally connected Darboux retract of $R^{2}$.

Under certain conditions we can show that a Darboux retract of a space is closed. A subset $A$ of a space $X$ satisfies condition $L$ if for each $x \in \bar{A}$ and neighborhood $U$ of $x$ in $X$ there exists a connected set $C$ such that $x \in \operatorname{bd}(C)$ and $C \subset A \cap U$.

ThEOREM 1. If $A$ is a Darboux retract of a Hausdorff space $X$ and $A$ satisfies condition $L$, then $A$ is closed.

Received by the editors December 11, 1977 and, in revised form, October 14, 1979.

AMS (MOS) subject classifications (1970). Primary 54C10.

$K e y$ words and phrases. Darboux retracts, condition $L$, absolute Darboux retract. 
Proof. Assume $x \in \bar{A}-A$. Let $r: X \rightarrow A$ be a Darboux retraction. Now $r(x) \in A$, so $x \neq r(x)$. Let $U$ and $V$ be disjoint neighborhoods of $x$ and $r(x)$, respectively. There exists a connected set $C$ such that $x \in \operatorname{bd}(C)$ and $C \subset A \cap U$. Thus $C \cup\{x\}$ is connected, but $r(C \cup\{x\})=C \cup\{r(x)\} \subset U \cup V$ is not.

THeOReM 2. If $r: X \rightarrow A$ is a Darboux retraction, $x \in \bar{A}-A$, and $U$ and $V$ are disjoint neighborhoods of $x$ and $r(x)$ respectively, then $x$ is not in the closure of any component of $U \cap A$.

Proof. Essentially the same as the proof of Theorem 1.

Corollary 1. If $A$ is a Darboux retract of $X$ and $x \in \bar{A}$ is such that $A \cup\{x\}$ is locally connected at $x$, then $x \in A$.

A space $X$ is locally peripherally connected if for each $x \in X$ and neighborhood $U$ of $x$, there exists a neighborhood $V$ of $x$ such that $\bar{V} \subset U$ and bd $(V)$ is connected.

THEOREM 3. If $r: X \rightarrow A$ is a Darboux retraction, where $X$ is connected and locally peripherally connected, then $r(\operatorname{bd}(A)) \subset \operatorname{bd}(A)$.

Proof. Assume $x \in \mathrm{bd}(A)$ and $r(x) \notin \mathrm{bd}(A)$. Then there exists a neighborhood $U$ of $r(x)$ such that $\bar{U} \subset \operatorname{int}(A)$ and $\operatorname{bd}(U)$ is connected. Then $A-U$ is connected by Theorem 60 of $[9$, p. 25]. Thus $(A-U) \cup\{x\}$ is connected, but $r((A-U) \cup$ $\{x\})=(A-U) \cup\{r(x)\}$ is not connected.

COROLlary 2. If $A$ is a Darboux retract of a connected, locally peripherally connected space $X$ and $A$ is a proper subset of $X$, then $A$ is not open.

That a compactum (compact metric space) $A$ is an absolute Darboux retract (ADR) means that if $A$ is a homeomorphic to a closed subset $A^{\prime}$ of a separable metric space $X$, then $A^{\prime}$ is a Darboux retract of $X$.

The following theorem is a special case of Theorem 3.2 of [7].

THEOREM 4. Let $A$ be a compactum. The following statements are equivalent:

(a) $A$ is an $A D R$.

(b) $A$ is homeomorphic to a Darboux retract of the Hilbert cube.

(c) If $f: X \rightarrow A$ is continuous, where $X$ is a closed subset of a separable metric space $X^{\prime}$, then there exists a Darboux function (called a Darboux extension) $f^{\prime}: X^{\prime} \rightarrow A$ such that $f^{\prime}(x)=f(x)$ for each $x \in X$.

THEOREM 5. Every metric continuum is an ADR.

Proof. It suffices to show that if $A$ is a metric continuum and $A \subset X$, where $X$ is separable metric, then $A$ is a Darboux retract of $X$. We define such a retraction on $X-A$ as a composition of functions.

For $x \in X$, let $d(x, A)=\inf d(x, y)$ over $y \in A$. Then $d$ is a continuous function from $X$ to the nonnegative real numbers and $d=0$ iff $x \in A$.

Let $\pi: R \rightarrow R / Q$ be the projection of the real numbers to the quotient group of the real numbers by the subgroup of rational numbers. 
If $\boldsymbol{A}$ is a point then a constant function would be a retraction. Suppose $\boldsymbol{A}$ has more than one point. Since $A$ is a connected separable metric space, $\operatorname{card}(A)=c$. Also, $\operatorname{card}(R / Q)=c$. Therefore there exists a 1-1 onto set mapping $h: R / Q \rightarrow A$.

For $x \in X-A$, let $r(x)=h(\pi(d(x, A)))$. For $x \in A$, let $r(x)=x$. This will be a Darboux retraction.

To show that $r$ is a Darboux function, let $K$ be a connected subset of $X$. If $K \subset A$, then $r(K)=K$ will be connected. If $K \subset X-A$ and $d(x, A)$ is constant for $x \in K, r(K)$ will be a point. If $K \subset X-A$ and $d(x, A)$ is not constant for $x \in K$, the image of $K$ under $d(x, A)$ will be a nondegenerate interval of real numbers. The function $\pi$ will send any such interval onto all of $R / Q$ and $h$ will send all of $R / Q$ onto all of $A$.

If $K$ has points in both $A$ and $X-A$, then $d(x, A)$ for $x \in K$ will range over an interval of nonnegative real numbers containing both 0 and at least one positive real number. The image of $K-A$ under $d(x, A)$ will contain all these numbers except zero, so it will be a nondegenerate interval. As above, $\pi$ will send this interval to all of $R / Q$ and $h$ will send that to all of $A$. Thus in all cases the image of $K$ is connected.

Corollary 3. Let $A$ be a subset of a separable metric space $X$ such that $A$ is connected. Then $A$ is a Darboux retract of $X$ if and only if $A$ is a Darboux retract of $\bar{A}$.

Theorem 6. Any subspace of a separable metric space $X$ which is a Peano space (compact, connected, locally connected) is a retract of $X$ by a Darboux function which is continuous except possibly at the boundary of the subspace.

Proof. We define such a retraction as a composition of functions. Let $A$ be a subspace of $X$ which is a Peano space. Let $d(x, A)=\inf d(x, y)$ over $y$ in $A$. Let $f_{i}$ : $[1 /(1+i), 1 / i] \rightarrow A$ be a continuous onto function which sends both the endpoints of the interval to a fixed chosen point of $A$. Let $f:(0, \infty) \rightarrow A$ be defined as follows: if $x \in[1 /(1+i), 1 / i]$, let $f(x)=f_{i}(x)$ and if $x>1$, let $f(x)$ be the fixed chosen point of $C$. The function $f$ is continuous.

For $x \in X-A$, let $r(x)=f(d(x, A))$, and for $x \in A$, let $r(x)=x$. Then $r$ is a retraction, and is separately continuous on $A$ and on $X-A$. The proof that $r$ is a Darboux function is similar to the proof in Theorem 5.

Theorem 6 also yields an extension theorem.

TheORem 7. Suppose $Y$ is a Peano continuum contained in an $A D R Y^{\prime}$ and $f$ : $X \rightarrow Y$ is continuous where $X$ is a closed subset of a separable metric space $X^{\prime}$. There exists a Darboux extension $f^{\prime}: X^{\prime} \rightarrow Y$ which is continuous except perhaps on $f^{-1}(\mathrm{bd}(Y))$, where $\mathrm{bd}(Y)$ is the boundary of $Y$ in $Y^{\prime}$.

Proof. By Theorem 6 there exists a Darboux retraction $r: Y^{\prime} \rightarrow Y$ which is continuous on $Y^{\prime}-\operatorname{bd}(Y)$. By 2.19 of [1, p. 103], there exists a continuous extension $g: X^{\prime} \rightarrow Y^{\prime}$ of $f$. Let $f^{\prime}=r g: X^{\prime} \rightarrow Y$.

EXAMPLE 3. An effectively defined retraction from the unit disk in $R^{2}$ onto its boundary is given. Let $t$ denote $\left(x^{2}+y^{2}\right)^{1 / 2}$. Then if $(x, y)$ is in the interior of the 
disk, let

$$
f(x, y)=(\cos (\pi /(1-t)), \sin (\pi /(1-t))) .
$$

For $(x, y)$ on the boundary of the disk, let $f(x, y)=(x, y)$. The proof of this example is similar to the proof of Theorem 5.

In [5] Hunt generalized the Borsuk-Ulam antipodal point theorem by showing that no connectivity function $f: S^{n} \rightarrow S^{n-1}$, where $S^{n}$ denotes the $n$-sphere, can commute with the antipodal map. We now show that this result does not extend to Darboux functions.

Example 4. We can regard $S^{2}$ as $D_{1} \cup D_{2}$, where $D_{1}$ and $D_{2}$ are disks, if we identify their boundary circles. Regard $S^{1}$ as the common boundary. Let $r$ : $D_{1} \rightarrow S^{1}$ be the retraction of Example 3. Define $f: S^{2} \rightarrow S^{1}$ as follows. If $x \in D_{1}$, let $f(x)=-r(x)$ denote the point antipodal to $r(x)$. If $x \in D_{2}$, let $-x \in D_{1}$ be the antipodal point and let $f(x)=r(-x)$.

\section{REFERENCES}

1. K. Borsuk, Theory of retracts, Monografie Mat., Tom 44, PWN, Warsaw, 1967.

2. A. M. Bruckner and J. G. Ceder, Darboux continuity, Jber. Deutsch. Math.-Verein. 67 (1965), 93-117.

3. J. L. Cornette, Connectivity functions and images of Peano contimum, Fund. Math. 75 (1966), 184-192.

4. J. L. Cornette and J. E. Girollo, Connectivity retracts of finitely coherent Peano continua, Fund. Math. 61 (1967), 177-182.

5. J. H. V. Hunt, A connectivity map $f: S^{n} \rightarrow S^{n-1}$ does not commute with the antipodal map, Bol. Soc. Mat. Méxicana 16 (1971), 43-45.

6. K. R. Kellum, Non-continuous retracts, Studies in Topology, N. Stavrakas and K. Allen, Eds., Academic Press, New York, 1975, pp. 255-261.

7. The equivalence of absolute almast continuous retracts and e-absolute retracts, Fund. Math. (to appear).

8. P. E. Long and W. D. Hammers, Certain non-contimuous retract functions, Math. Chronicle 4 (1976), 122-140.

9. R. L. Moore, Foundations of point set theory, rev. ed., Amer. Math. Soc. Colloq. Publ., Vol. 13, Amer. Math. Soc., Providence, R. I., 1962.

Departugant of Mathematics, Alabama State Universtity, Montgomirgy, Alabaya 36101

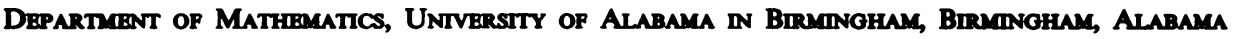
35294 